\title{
Uso del fertilizante (15-20-25) en la etapa de floración de Kalanchoe blossfeldiana
}

Use of the fertilizer (15-20-25) in the blooming stage of Kalanchoe blossfeldiana

\author{
Noriega Lucio Ana Cristina ${ }^{1}{ }^{\bowtie}$ López Castro Darío; Rodríguez Cabrera, Rocío ${ }^{1}$; Nieto Rosaliano Sergio Omar ${ }^{1}$ y \\ Elorza Martínez Pablo ${ }^{1}$ \\ ${ }^{1}$ Universidad Veracruzana. Facultad de Ciencias Biológicas y Agropecuarias campus Tuxpan
}

\utor para correspondencia: anyta piscis 10@hotmail.com

Recibido: 15/07/2017

Aceptado: 10/12/2017

\section{RESUMEN}

Se evaluaron 4 dosis del fertilizante 15-20-25 fueron 2, 3, 4 y 5 gramos por litro de agua más un testigo. El objetivo fue Determinar la mejor dosis de 15-20-25 para favorecer la floración de Kalanchoe blossfeldiana. Los resultados mostraron que la variable Contenido de clorofila a los 90 días el mejor tratamiento fue el No. 4, con54.95 unidades SPAD, con una diferencia significativa del tratamiento 5 que fue el testigo con 44.64. En la variable de Diámetro de tallo el tratamiento que tuvo mejor resultado fue el tratamiento 2 con 0.741 centímetro, teniendo una diferencia significativa con el testigo. En la variable de número de hojas, el Tratamiento que tuvo mayor número fue el 4 con un total de 20 hojas. En este sentido vemos que el tratamiento que funcionó de mejor manera y que obtuvo mayor contenido de clorofila, mayor diámetro de tallo y numero de hojas fue el tratamiento No. 4, con una dosis de 5 gramos por litro, donde se pudo observar plantas vigorosas de hojas gruesas y color verde obscuro por el alto contenido de nitrógeno registrado en la planta siendo además las que primero iniciaron la etapa de floración y mejor calidad de ramo floral.

Palabras clave: Floración, Kalanchoe, Fertilizante.

\begin{abstract}
ABSTRAC
Four doses of the fertilizer were evaluated. 15-20-25 were 2, 3, 4 and 5 grams per liter of water plus a control. The objective was to determine the best dose of 15-20-25 to favor the flowering of Kalanchoe blossfeldiana. The results showed that the variable Chlorophyll content at 90 days the best treatment was No. 4, with 54.95 units SPAD, with a significant difference of treatment 5 that was the control with 44.64. In the stem diameter variable, the treatment that had the best result was treatment 2 with $0.741 \mathrm{~cm}$, having a significant difference with the control. In the variable number of leaves, the Treatment that had the highest number was 4 with a total of 20 leaves. In this sense we see that the treatment that worked best and that obtained higher chlorophyll content, larger stem diameter and number of leaves was treatment No. 4, with a dose of 5 grams per liter, where vigorous plants could be observed of thick leaves and dark green color due to the high nitrogen content registered in the plant, being also those that first started the flowering stage and better quality of floral bouquet.
\end{abstract}

Keywords: Flowering, Kalanchoe, Fertilizer. 


\section{INTRODUCCIÓN}

En México la actividad ornamental es una actividad practicada desde hace mucho tiempo, principalmente en celebraciones rituales y festividades. Actualmente la producción de flores ha tomado importancia económica, estimándose que existen 14400 hectáreas cultivadas con flores, de las cuales el $90 \%$ se concentra en cinco estados de la república mexicana. La producción se destina al mercado local y solo un $10 \%$ a la exportación (SAGARPA, 2009).

La familia Crassulaceae resalta en el territorio mexicano por sus más de 300 especies conocidas en el país, lo cual lo ubican en el primer lugar a escala mundial por su número de taxa, el segundo lugar le corresponde a Sudáfrica con cerca de 225 especies (Thiede, 1995). Esta familia está conformada por plantas suculentas, la mayoría de los miembros muestran preferencia por sitios con afloramientos rocosos; se encuentra ampliamente distribuida en el mundo, exceptuando Australia (de donde se conoce sólo una especie de Tillaea) y Polinesia (de donde no se tienen registros); se localizan miembros de ella casi en cualquier parte de la Tierra. Sin embargo, se han identificado algunas regiones en las cuales este grupo ostenta mayor diversidad, como son la zona centro-sur de Asia, Sudáfrica, el Mediterráneo y México (Ordoñez y Briones, 2004). El objetivo de la investigación fue Evaluar el efecto del fertilizante finalizador 15-20-25 en el desarrollo de la planta de Kalanchoe blossfeldiana. El Kalanchoe blossfeldiana (Poelln.) pertenece a la familia de las Crasuláceas, se desarrolla a una temperatura promedio de $15{ }^{\circ} \mathrm{C}$ de primavera a verano a una altitud de $2000 \mathrm{~m}$, dentro de un ambiente templado y sombreado a diferencia de los otros miembros de dicha familia que habitan en áreas secas y soleadas, con hojas gruesas, suculentas adaptadas a ambientes desérticos. Se le clasifica como una planta de día corto, la mayoría de los cultivares requieren de 14 a $15 \mathrm{~h}$ de obscuridad continua para desarrollar flores durante cuatro a cinco semanas. El crecimiento de las hojas varía en un día corto o largo, en el primero las hojas son gruesas, suculentas y rígidas, mientras que en el segundo son delgadas y flexibles. (William, 1988). En México la oferta en el sector florícola es inferior a la demanda, y en América, países como Estados Unidos y Canadá, presentan cada vez mayor interés en este sector, sin embargo la competencia internacional es un factor importante: Colombia, Ecuador y Costa Rica compiten con los productos mexicanos en calidad y cantidad. En el resto del mundo, Holanda es el país líder en materia florícola y tecnología, aunque se destacan también países como Israel, Kenia y Alemania (CONMEXFLOR, 2006).

Para 1994, con la entrada en vigor del Tratado de Libre Comercio de América del Norte (TLCAN) se inició una nueva etapa del comercio internacional en México. El sector de la floricultura se enfrentó con problemas como el desconocimiento de los requisitos para

exportar, la infraestructura existente incompetente, o la reglamentación vigente en el mercado que, aunados a la política de desgravación arancelaria, impidieron el crecimiento proyectado para los años siguientes al TLCAN (García, 1999). Del total de la producción florícola mexicana, un $90 \%$ se destina al mercado doméstico y sólo el $10 \%$ al mercado de exportación. Actualmente, el $8 \%$ de esta producción se cultiva en invernadero y el $92 \%$ a cielo abierto (Usabiaga, 2006). El calcio es absorbido por las plantas en su forma catiónica Cay es parte constituyente de las sales en la solución del suelo. En el interior de la planta es un elemento poco móvil interviniendo en la forma de los pectatos de calcio de la laminilla media de las células que intervienen en el proceso de absorción de los elementos. El calcio forma sales con los ácidos orgánicos e inorgánicos del interior de las células regulando la presión osmótica de la misma. Interviene en la formación de la lecitina, que es el fosfolípido importante en la membrana celular, siendo un 
factor importante en la permeabilidad de estas membranas. Igualmente actúan en la división mitótica de las células, en el crecimiento de los meristemos y en la absorción de nitratos (Rodríguez, 1992).

La calidad de las plantas ornamentales en maceta depende, fundamentalmente, del tipo de sustrato que se utilice para cultivarlas y, en particular, de sus características físico-químicas, ya que el desarrollo y el funcionamiento de las raíces están directamente ligados a las condiciones de aireación y contenido de agua, además de tener una influencia directa sobre el suministro de nutrimentos necesarios para las especies que se desarrollen en él. Todas estas interacciones se reflejarán positiva o negativamente en la presentación comercial final de las especies cultivadas (Bunt, 1988).

\section{MATERIALES Y MÉTODOS}

$$
\text { El experimento se estableció }
$$

Facultad de Ciencias Biológicas y Agropecuarias de la Universidad Veracruzana en el Municipio de Tuxpan Ver, (2057' 10.16”N, $97^{\circ} 27^{\prime} 21.73$ " O; $10 \mathrm{msnm}$ ); el clima de la región es tropical, con una precipitación media anual de $1241 \mathrm{~mm}$; lluvias abundantes en verano, y principios de otoño con menor intensidad de noviembre a mayo con una precipitación promedio anual de $24{ }^{\circ} \mathrm{C}$. Se utilizó el diseño experimental bloques completos al azar, con 5 tratamientos y 3 repeticiones, se evaluó el fertilizante finalizador (15-20-25) con cuatro dosis diferentes 2.0, 3.0, 4.0 y 5.0 gramos por litro de agua, más un testigo, originando 5 tratamientos en total. El diseño experimental utilizado fue Bloques completos al azar con 5 tratamientos y 4 repeticiones tienen 4 unidades experimentales y cada una de ellas esta constituidas por una maceta de 6 pulgadas, Para el procesamiento de los datos se utilizó el paquete estadístico Statistica. Se utilizó un sustrato a base de materia orgánica y arena, el cual se preparó revolviéndolo uniformemente, el trasplante de los esquejes se realizó de manera manual, llenando primero cada una de las macetas con la misma proporción de sustrato. Posteriormente se colocó un esqueje en cada maceta. El fertilizante que se utilizó es un finalizador de concentración (15-20-25) de la marca PETERS con diferentes dosis para cada tratamiento, el cual se pesó el fertilizante para cada tratamiento esto se realizó en el laboratorio de suelos utilizando una báscula digital, pasando primero 2 gramos: para el Tratamiento 1, 3 gramos: para el Tratamiento 2, 4 gramos: para el tratamiento 3 y 5 gramos: para el tratamiento 4, se empezó aplicar a los 30 días antes del fotoperiodo de la planta y sucesivamente hasta la etapa de la floración. El riego en este cultivo fue controlado, ya que esta planta no tolera el exceso de humedad. Por tal motivo el riego se realizó solo dos veces por semana aplicando la dosis que corresponde a cada tratamiento junto con el agua de riego.

\section{RESULTADOS}

Los resultados muestran el comportamiento en cada uno de las plantas de Kalanchoe blossfeldiana con las dosis de fertilización. En la variable contenido de

clorofila, se encontró diferencia estadísticamente significativa para una probabilidad del $5 \%$ entre el mejor tratamiento que fue el 4 con 53.03 unidades con una dosis de fertilización de 5 gramos por litro y el tratamiento número 2 con 50.7 con una dosis más baja de 3 gramos por litro. Esto porque el nitrógeno en condiciones optimas se produce mayor actividad fotosintética, ya que este forma parte de la estructura de la clorofila (Rodríguez, 1989).

En un trabajo realizado para estimar la concentración de nitrógeno y clorofila en tomate con uso de un SPAD, menciona que la concentración de clorofila mostro valores conforme aumenta la concentración de nitrógeno (Rodríguez, 1989). En la variable Diámetro de tallo el tratamiento que obtuvo mejores resultados fue el tratamiento 2 con .741 centímetro, teniendo una diferencia significativa del $5 \%$ con nuestro testigo de $0.591 \mathrm{~cm}$. La 
variable número de hojas nos muestra que el tratamiento que obtuvo mayor cantidad de hojas fuel Tratamiento 4 con un promedio de 20 hojas desarrolladas. Seguida del tratamiento 2 con un promedio de 19 hojas. Según Tso (1990) la reducción del número de hojas por planta trae aparejado un incremento en el proceso fotosintético, que provoca que las sustancias elaboradas por la planta se distribuyan en menor cantidad de hojas, por lo tanto, aumentan la longitud, anchura y materia seca de ellas. Todo lo contrario, sucede cuando el número de hojas dejadas al desbotonar aumenta. Finalmente se observó que el tratamiento que mejores resultados presentó con respecto a los parámetros de floración fue el 4, mismo que presento uniformidad y la mejor coloración de las flores.

\section{CONCLUSIÓN}

El contenido de clorofila muestra que el tratamiento No. 4 es el que mejor asimiló los fertilizantes, esto debido a que el contenido de clorofila nos referencia el contenido de nitrógeno en la planta, lo que es importante para favorecer el crecimiento después de los pinchados. Mientras que el tratamiento No. 2 igualmente fue el mejor para la variable de respuesta diámetro de tallo, lo que nos muestra una mejor adaptación a las condiciones donde se realizó el experimento. En la variable de número de hojas el tratamiento con mayor cantidad de hojas fue el T4 con un aproximado de 20 hojas.

Por lo anterior se concluye que el mejor tratamiento en consideración de la influencia que tuvo la fertilización fue el 4 donde se aplicaron $5 \mathrm{gr} / \mathrm{lt}$ de agua. Mostrando diferencias estadísticamente significativas con respecto a los tratamientos $1,2,3 \mathrm{y}$ al testigo.

\section{LITERATURA CITADA}

Alfaro O. R 2015. Fertilidad de Suelos y Nutrición De Plantas. 15/06/2017, de UNIVERSIDAD
RAFAEL LANDIVAR SEDE ESCUINTLA

Sitio web:

file:///F:/Conceptos_de_fertilidad_2015_parte _1.pdf

Ávila, S. M.; G. De La Rosa A.; Reyes, S. J. 2005. Jardín Botánico del Instituto de Biología de la UNAM, La colección de crasuláceas (siemprevivas). Floral. XV (1): $14-16$

BallSeed. 1992. Plant catalog 1992-1993. Geo. J. Ball. Inc. pp 12-13 y 52-53.

Bowman, D.C.; Paul, J.L. 1983. Understanding of container media vital knowledge for growing successful plants. Pacific Coast Nursery man and Garden Supply Dealer. MarchIssue.

Bunt, A.C. 1988. Media and mixes forcontainergrownplants. UnwinHyman. London, Great Britain https://doi.org/10.1007/978-94-011-7904-1

Cronquist, A. 1981. Introducción a la Botánica. Compañía Editorial Continental. México.

Davidson, H.; Peterson, C.; Mecklenburg, R. 1994. Nursery Management: Administation and

Culture. 2nd. Ed. Prentice Hall Career\&Technology, New Jersey, USA. 486 p.

Farnham, D.S.; Hasek, R.F.; Paul, J.L. 1985. Waterquality; Itseffectson ornamental plants. CooperativeExtensionLeaflet No. 2995. University of California, USA. 15 p.

Gehrig H Gaussmannm O Marx H Schwarzott D y M Kluge. 2001. Molecular de la filogenia género Kalanchoe (Crassulaceae) deducido a partir de secuencias de nucleótidos de las ITS-1 y sus regiones-2.Plant Sciences. 160. 827-835. https://doi.org/10.1016/S0168-9452(00)00447-7

Hartmann, H.T. y D.E. Kester. 1992. Propagación de plantas. Compañía Editorial Continental, S.A. de C.V., México. pp 137-215

Jiménez, M. R. y R. M. Caballero. 1990. El cultivo industrial de plantas en o Maceta. Ediciones de Horticultura, S.L. Reus. España. 
Marschenr, H1995. Mineral Nutrition of

HigherPlants. Secindseditioninstitute of

PlantNutritionUniversity

HohenheimGermany. HarcountBrace and CompanyPublishers. London.

Martínez M., F. 1994. Manual básico de sustratos. Oasis Consultoria. Jiutepec, Mor.

Meyrán, J., y L. López. 2003. Las Crasuláceas de México. Sociedad Mexicana de Cactología, A.C. México, D.F.234 pp

Nelson, P.V. 1991. Greenhouse Operation and Management. 4th Edition. Prentice Hall, Englewood Cliffs, NJ, USA. 611 p.

Pertuit, A.J. 1992. Introduction to floricultura. Second Edition. Academic Press, Inc. USA.
Rodríguez, S.A 1992. Fertilizantes, Nutrición Vegetal. AGT edición, segunda reimpresión. México, DF.

SAGARPA, 2009, Plan Rector Sistema Producto Ornamentales Puebla.

Tso, T. C.: Production, Physiology, andBiochemistry of TobaccoPlant, 753 pp., Ed. Institute of International Development and Education in Agricultural and LifeSciences, USA, 1990

Velázquez R., J.M. 1995. Evaluación de Quercus crassipes en vivero bajo diferentes tipos de sustrato e intensidades de luz. Tesis profesional. Universidad Autónoma Chapingo. Chapingo, México

Copyright (c) 2017 Ana Cristina Noriega Lucio, Dario López Castro, Rocio Rodriguez Cabrera,

Sergio Omar Nieto Rosaliano y Pablo Elorza Martinez

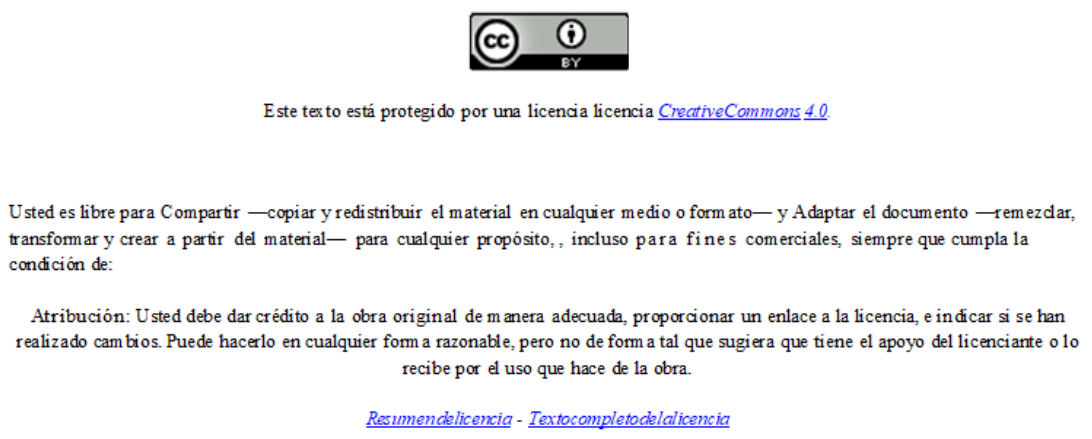

